\title{
Endothelial protein C receptor-associated invasiveness of rheumatoid synovial fibroblasts is likely driven by group $V$ secretory phospholipase $A_{2}$
}

Meilang Xue ${ }^{1 *}$, Kaitlin Shen ${ }^{1}$, Kelly McKelvey ${ }^{1}$, Juan Li ${ }^{1}$, Yee-Ka Agnes Chan ${ }^{1}$, Vicky Hatzis ${ }^{1}$, Lyn March$^{2}$, Christopher B Little ${ }^{3}$, Michael Tonkin ${ }^{4}$ and Christopher J Jackson ${ }^{1}$

\begin{abstract}
Introduction: Rheumatoid synovial fibroblasts (RASFs) mediate joint inflammation and destruction in rheumatoid arthritis (RA). Endothelial protein $C$ receptor (EPCR) is a specific receptor for the natural anticoagulant activated protein C (APC). It mediates the cytoprotective properties of APC and is expressed in rheumatoid synovial tissue. A recent report shows that group $V$ secretory phospholipase $A 2\left(s P L A_{2} V\right)$ prevents $A P C$ from binding to EPCR in endothelium and inhibits EPCR/APC function. The aim of this study was to investigate the expression and function of EPCR on RASFs.
\end{abstract}

Methods: Human synovial fibroblasts (SFs) were isolated from RA or osteoarthritis (OA) synovial tissues and treated with control, EPCR, or $S P L A_{2} V$ small interfering RNA (siRNA); recombinant human APC, tumor necrosis factor-alpha (TNF-a), or sPLA ${ }_{2}$. RASF viability and migration/invasion were measured by 3-[4,5-dimethylthiazol-2-yl]-2,5-diphenyl tetrazolium bromide (MTT) and collagen gel migration/invasion assays, respectively, and cartilage degradation by 1,9-dimethylmethylene blue (DMMB) assay in the presence of human OA articular cartilage explants. The expression or activation of cytokines, EPCR, cadherin-11, mitogen-activated protein (MAP) kinases, and nuclear factor-kappa-B (NF-kB) or both were detected by enzyme-linked immunosorbent assay, Western blotting, or immunostaining.

Results: EPCR was expressed by both OASFs and RASFs but was markedly increased in RASFs. When EPCR was suppressed by siRNA or blocking antibody cell viability, cell invasion and cartilage degradation were reduced by more than 30\%. Inflammatory mediators interleukin-1-beta (IL-1ß), cadherin-11, and NF-KB were significantly reduced by EPCR suppression under control or TNF-a-stimulated conditions. The expression or activation (or both) of MAP kinases ERK, p38, and JNK were also markedly decreased in cells transfected with EPCR siRNA. Further analysis revealed that $S P L A_{2} \vee$ co-localized with EPCR on RASFs. Suppression of $S P L A_{2} V$ reduced cell viability and cartilage degradation and increased APC binding to RASFs. Conversely, recombinant sPLA $\mathrm{A}_{2} \mathrm{~V}$ increased cartilage degradation, blocked APC binding to RASFs, and could not rescue the effects induced by EPCR suppression.

Conclusions: Our results demonstrate that EPCR is overexpressed by RASFs and mediates the aggressive behavior of RASFs. This function of EPCR is contrary to its cytoprotective role in other settings and is likely driven by sPLA $\mathrm{A}_{2}$.

\footnotetext{
* Correspondence: meilang.xue@sydney.edu.au

${ }^{1}$ Sutton Arthritis Research Laboratories, The University of Sydney at Royal North Shore Hospital, Pacific Highway, St Leonards, NSW 2065, Australia Full list of author information is available at the end of the article
} 


\section{Introduction}

Rheumatoid arthritis (RA) is a chronic inflammatory disease characterized by synovial inflammation and hyperplasia, leading to progressive cartilage and bone destruction. Normal synovium forms a thin membrane at the edges of joints and provides lubrication and nutrients for the cartilage. In RA, this thin synovial lining layer dramatically increases and transforms into an inflammatory mass, known as the pannus [1,2]. This tissue mass expands and attaches to and invades the adjacent cartilage and subchondral bone, causing erosion. The major cell type accounting for the thickened lining layer and resultant pannus is the activated RA synovial fibroblasts (RASFs, also referred to as RA synoviocytes). As well as mediating tissue destruction, RASFs play a major role in catalyzing and sustaining RA by producing inflammatory cytokines such as interleukin-1-beta (IL-1 $\beta$ ) and tumor necrosis factoralpha (TNF- $\alpha$ ), proangiogenic factors, and matrix-degrading enzymes $[1,2]$. Of equal concern, RASFs collaborate with and support the recruitment, survival, activation, and differentiation of T cells, B cells, macrophages, mast cells, osteoclasts, and endothelial cells throughout the RA synovium $[1,2]$.

Once activated, the aggressive phenotype of RASFs can exist independent of inflammation. This was demonstrated by studies conducted in the severe combined immunodeficient mouse model of RA, in which implanted human RASFs degraded co-implanted human cartilage in the absence of inflammatory cells [3], and RASFs migrated via the bloodstream to implanted cartilage at a distant site, spreading RA to unaffected joints [4]. These data clearly indicates that RASFs are not passive bystanders, but are active participants in joint destruction in RA.

Endothelial protein $C$ receptor (EPCR) is an endothelial transmembrane glycoprotein able to bind to a natural anticoagulant, protein $\mathrm{C}$ (PC), and its activated form, APC, with similar affinity [5]. Though originally identified as an endothelial cell receptor, EPCR has since been detected on many other cell types [6], including RA synovial lining cells [7]. As a receptor, EPCR mediates the majority of the anti-apoptotic, anti-inflammatory, and barrierprotective functions of APC [8]. In addition, EPCR itself is a central player in the convergent pathways of homeostasis and inflammation [8]. Recently, EPCR has been found to be overexpressed by some cancer cells and increased cancer cell migration and invasion [9-11]. However, the underlying mechanisms are not clear. EPCR can be cleaved from the cell surface to form soluble EPCR (sEPCR), which binds PC/APC with the same affinity as membrane-bound EPCR but blocks the protective function of APC [12-14]. Increased sEPCR is associated with many inflammatory/autoimmune diseases [15-17]. A recent report shows that group V secretory phospholipase
A2 (sPLA 2 V) prevents APC binding to EPCR and inhibits EPCR/APC function by accommodating lysophosphatidylcholine (LysoPCh) or platelet-activating factor (PAF) in the hydrophobic groove of EPCR [18]. Being a key enzyme in the production of diverse mediators of inflammatory conditions, $\mathrm{sPL}_{2} \mathrm{~V}$ is present in significantly higher levels in the RA joint $[19,20]$ and stimulates RA synovial proliferation and joint destruction [20,21].

The role of EPCR has not been studied in RASFs. We report here that EPCR is highly expressed by RASFs and contributes to the inflammatory and cartilage-degradative actions of these cells in vitro. Importantly, we show that these unexpected destructive effects of EPCR are likely driven by $\mathrm{SLA}_{2} \mathrm{~V}$.

\section{Methods and materials}

Fetal bovine serum (FBS), anti-cadherin 11 antibody, and TRIzol were purchased from Invitrogen (Carlsbad, CA, USA); anti-ERK, phosphorylated ERK (P-ERK), p38, P-p38 antibodies and anti-fibroblast marker (ER-TR7), A/G Plus-agarose, scrambled control, validated EPCR, $\mathrm{PC}$, and $\mathrm{sPLA}_{2} \mathrm{~V}$ small interfering RNAs (siRNAs) from Santa Cruz Biotechnology (Santa Cruz, CA, USA); recombinant APC from Eli Lilly and Company (Indianapolis, IN, USA); RiboCellin Transfection Reagents from BioCellChallenge (Paris, France); anti- $\beta$-actin and PC/APC antibodies and recombinant TNF- $\alpha$ from Sigma-Aldrich (St. Louis, MO, USA); anti-human CD68 from eBioscience (San Diego, CA, USA); human IL-1 $\beta$, IL-6, and IL-8 enzyme-linked immunosorbent assay (ELISA) DuoSet from R\&D Systems (Minneapolis, MN, USA); anti-nuclear factor-kappa B (anti-NF- $\mathrm{kB}$ ) p65 subunit antibody from Chemicon International (Temecula, CA, USA); recombinant sPLA ${ }_{2} \mathrm{~V}$ from Abcam (Cambridge, MA, USA); antihuman $\mathrm{sPLA}_{2} \mathrm{~V}$ antibody from Cayman (Ann Arbor, MI, USA); NE-PER nuclear extraction reagents from Pierce Chemical Co. (Rockford, IL, USA). RCR252 (EPCR blocking antibody) and RCR92 (EPCR non-blocking antibody) were kindly provided by Kenji Fukudome (Saga Medical School, Saga, Japan).

\section{Synovial fibroblast isolation, culture, and treatment}

Human SFs were isolated from synovial tissues obtained from knee replacement surgeries of seven patients with RA and eight patients with osteoarthritis (OA) (Table 1).

Table 1 Demographics of patients from which synovial fibroblasts were isolated from synovial tissues obtained from knee replacement surgeries

\begin{tabular}{lccccc}
\hline & \multicolumn{2}{c}{ Osteoarthritis } & & \multicolumn{2}{c}{ Rheumatoid arthritis } \\
\cline { 2 - 3 } & Female & Male & & Female & Male \\
\hline Patients, number & 3 & 5 & & 5 & 2 \\
Age, years & $69.2 \pm 7.2$ & $71 \pm 5.7$ & & $68.1 \pm 6.2$ & $70.5 \pm 4.7$ \\
\hline
\end{tabular}


After collagenase digestion, SFs were collected and cultured in Dulbecco's modified Eagle's medium (DMEM) supplemented with $10 \%$ FBS. Purity of RASFs was determined by flow cytometry stained with anti-CD68 antibody (eBioscience) and anti-fibroblast marker (ER-TR7). Cells were used for further experiments if more than 95\% cells were positive for fibroblast marker. Passage 1 to 3 cells were used in all experiments. Confluent cells in 24-well plates were serum-starved with DMEM without FBS for 4 hours. After replacement with fresh DMEM, cells were treated with different reagents for 24 hours. Cells and culture supernatants were collected for further analysis. There was no difference in cell viability, detected by trypan blue exclusion and 3-[4,5-dimethylthiazol-2yl]-2,5-diphenyl tetrazolium bromide (MTT) assays (see below for details), in response to different treatments for 24,48 , or 72 hours at the concentrations used for this study, compared with no treatment controls (data not shown).

\section{Synovial fluid and cartilage}

Synovial fluid samples were collected by needle puncture from the knee joints of 12 patients with RA and 12 patients with OA (Table 2). OA cartilage was obtained from knee/hip replacement surgeries.

Usage of human tissue and fluid samples was in accordance with the ethics committee of the Northern Sydney Local Health District. All patients fulfilled the American College of Rheumatology criteria for RA and OA [22,23] and gave their written informed consent.

\section{siRNA transfection}

SFs were transfected with EPCR, sPLA 2 , or scrambled control siRNAs using RiboCellin Transfection Reagent in accordance with the protocol of the manufacturer. The efficacy of siRNA was detected by reverse-transcription real time polymerase chain reaction, ELISA, or Western blot.

\section{ELISA}

EPCR, IL-1 $\beta$, IL-6, and IL- 8 in culture supernatants/ whole cell lysates or synovial fluids were measured by ELISA DuoSet in accordance with the instructions of the manufacturer.

Table 2 Demographics of patients from which synovial fluid samples were collected by needle puncture from the knee joints

\begin{tabular}{lccccc}
\hline & \multicolumn{2}{c}{ Osteoarthritis } & & \multicolumn{2}{c}{ Rheumatoid arthritis } \\
\cline { 2 - 3 } & Female & Male & & Female & Male \\
\hline Patients, number & 9 & 3 & & 5 & 7 \\
Age, years & $68.2 \pm 5.1$ & $70 \pm 4.5$ & & $67.0 \pm 4.6$ & $69.5 \pm 3.6$ \\
\hline
\end{tabular}

\section{Gelatin zymography}

Matrix metalloproteinase-2 (MMP-2) and MMP-9 protein secretion and activation in the culture supernatants were measured by using gelatin zymography under nonreducing conditions [24].

\section{Immunoprecipitation and Western blot}

SFs were lysed in cell lysis buffer (20 mM HEPES, 1\% Triton X, and 10\% Glycerol) supplemented with protease and phosphate inhibitors. Nuclear proteins were extracted with NE-PER nuclear extraction reagents in accordance with the instructions of the manufacturer. Immunoprecipitation (IP) was performed by using A/G Plus-agarose after incubation with anti-human EPCR antibody. Equal amounts of protein were separated on $10 \%$ or $15 \%$ (for IP) SDS-PAGE. Immunoreactivity was detected by using the ECL detection system and semiquantified with gel image analysis software. Anti-human $\beta$-actin antibody was used to normalize equal loading.

\section{In vitro migration/invasion assay}

RASF invasion was measured by using a collagen gel assay [25] with modifications. Briefly, RASFs $\left(2 \times 10^{5}\right.$ cells/mL in DMEM) were mixed with equal volume of collagen solution (2 mg/mL, pH 7.2) and transferred into 24well plates ( $20 \mu \mathrm{L} /$ drop, 4 drops/well). After polymerization for 2 hours, $0.5 \mathrm{~mL}$ DMEM with $10 \%$ FBS was added to each well, and plates were incubated at $37^{\circ} \mathrm{C}$. After 48 hours, cells and collagen gels were stained with crystal violet. Cells that had migrated out of gels were counted under a light microscope. At least 16 collagen gel drops per experimental group were analyzed.

\section{Immunohistochemistry, immunofluorescence,} and toluidine blue staining

Cultured SFs in Falcon culture slides (Becton, Dickinson and Company, Franklin Lakes, NJ, USA) were fixed with $1 \%$ paraformaldehyde. Human synovial tissues were fixed in $10 \%$ phosphate-buffered saline-buffered formalin. Immunohistochemical and immunofluorescent staining and toluidine blue staining were performed as described previously [26,27]. Isotype IgG for each antibody was used as a negative control. Images were acquired and processed by using a digital camera and software (Nikon, Tokyo, Japan) and ImageJ.

\section{MTT assay}

Cells $\left(4 \times 10^{3} /\right.$ well $)$ were seeded into a 96-well plate. After 4 hours' attachment, cells were treated with different test agents for 24,48 , or 72 hours, and cell viability was detected by the colorimetric MTT assay and confirmed by a trypan blue exclusion test. 
1,9-dimethylmethylene blue assay

The level of sulphated glycosaminoglycans (sGAGs) released from the cartilage explants was determined by a 1,9-dimethylmethylene blue (DMMB) assay against a standard curve of chondroitin sulfate [28].

\section{Statistical analysis}

Significance was determined by using one-way analysis of variance followed by Tukey's honestly significant difference (HSD) post hoc test or Student $t$ test. $P$ values of less than 0.05 were considered statistically significant.

\section{Results}

\section{EPCR is overexpressed by RASFs}

ECPR expression by synovial tissues was determined by immunostaining. There was a stronger staining in RA synovial tissue than in OA tissue (Figure 1A). Most EPCR staining was localized to the lining and sub-lining layers. As expected, blood vessels were positively stained for EPCR. Interestingly, there was no difference in the levels of sEPCR, which could bind PC/APC and inhibit the function of APC [14], in synovial fluids from RA versus OA patients (Figure 1B).

To confirm whether SFs express EPCR, dual immunofluorescent staining was performed by using anti-EPCR antibody and the fibroblast marker, ER-TR7. EPCR staining in the lining layer was localized on SFs in both OA and RA synovium (Figure 1C). Isolated RASFs also expressed higher levels of EPCR than OASFs as assessed by immunostaining (Figure 1D). ELISA data using whole cell lysates confirmed that RASFs expressed threefold higher EPCR than OASFs (Figure 1E). sEPCR was not detectable in culture supernatants of RASFs or OASFs. At the gene level, both OASFs and RASFs expressed EPCR mRNA, with
A

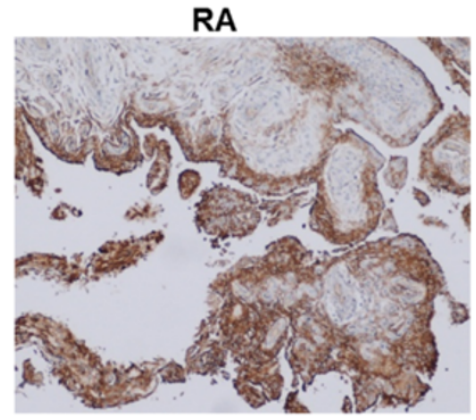

\section{OA}

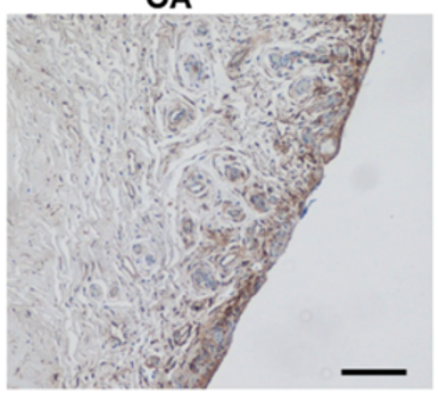

B

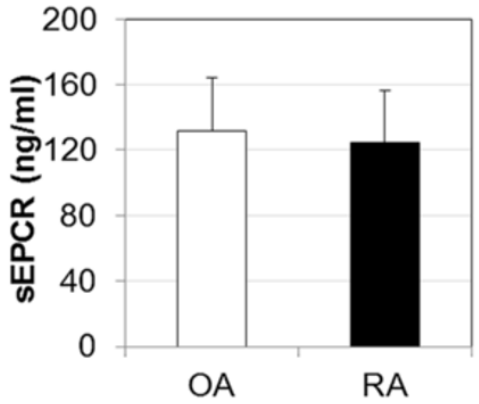

C

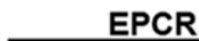

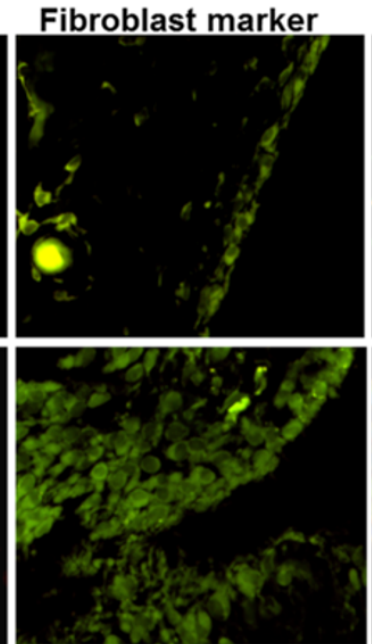

Merged

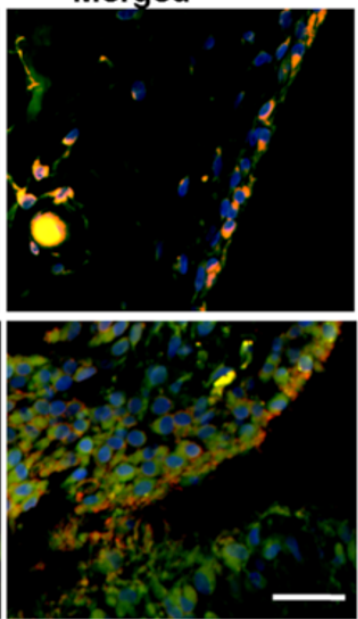

D
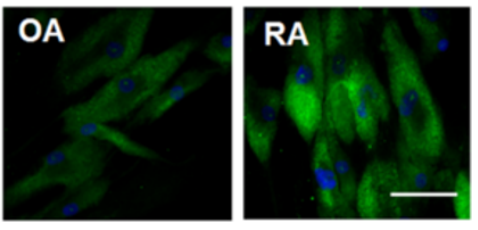

E

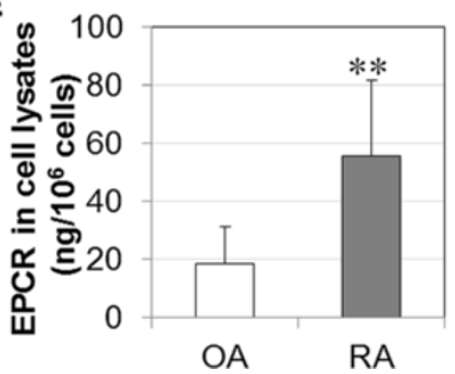

Figure 1 Expression of endothelial protein C receptor (EPCR) in synovial tissues, synovial fluids, and cultured synovial fibroblasts (SFs). (A) EPCR expression in synovial tissues from patients with rheumatoid arthritis (RA) and osteoarthritis (OA), detected by immunohistochemistry, and counterstained with Hematoxylin and Scott's Bluing Solution. Scale bar: $400 \mu \mathrm{m}$. (B) Soluble EPCR (sEPCR) in synovial fluids from patients with OA and RA, detected by enzyme-linked immunosorbent assay (ELISA) and expressed as mean \pm standard deviation (SD) ( $n=12$ each). (C) Co-localization of EPCR with the fibroblast marker, ER-TR7, in synovial tissues from patients with RA and OA, detected by immunofluorescent staining. Scale bar: $50 \mu m$. (D) EPCR expression in cultured SFs, detected by immunofluorescent staining. Scale bar: 20 um. Nuclei were counterstained with 4'-6-diamidino-2phenylindole (DAPI) (blue) in (C) and (D). The images represent one of three experiments. (E) EPCR in whole cell lysates of cultured SFs detected by ELISA and expressed as mean \pm SD ( $n=7$ each). Data was analyzed by Student $t$ test, ${ }^{* *} P<0.01$. 
RASFs expressing more than 50\% higher levels than OASFs at passage 1 .

\section{Suppressing EPCR inhibits the aggressive properties of RASFs}

To examine whether EPCR is associated with the aggressive properties of RASFs, EPCR expression was suppressed by its specific siRNA or function was blocked by the blocking antibody RCR252. The efficacy of siRNA was confirmed by reverse transcription-polymerase chain reaction and ELISA, which suppressed gene expression by more than $85 \%$ at 24 hours and protein levels by more than $75 \%$ at 72 hours after transfection (Figure 2A,B).
RASF survival/growth, a critical step that leads to synovial hyperplasia, was measured at 72 hours after transfection. Suppressing EPCR with siRNA resulted in a more than $30 \%$ reduction in RASF viability (Figure 2C). This was confirmed by the blocking antibody RCR252 treatment, which displayed a significant reduction in viable cells, whereas the non-blocking control antibody, RCR92, had no effect (Figure 2C). Another aggressive property of RASFs is their elevated migratory/invasive capacity. To assess this, a collagen gel migration/invasion assay was performed. In basal conditions, $20 \%$ to $30 \%$ of cells migrated out of a gel after 48 hours of incubation. There were more than $30 \%$ fewer cells migrating out of the collagen gel
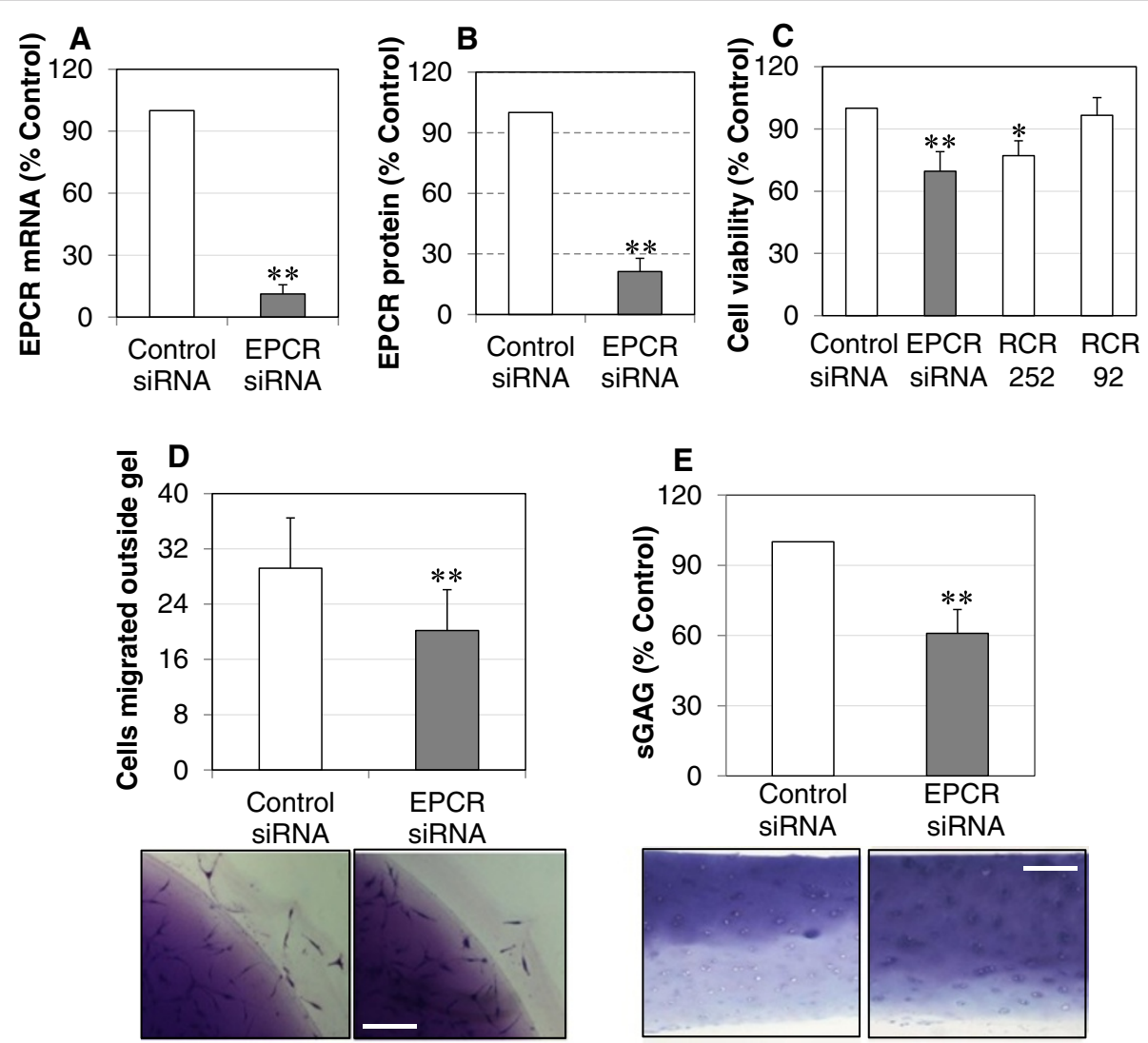

Figure 2 Blocking endothelial protein C receptor (EPCR) suppresses rheumatoid synovial fibroblast (RASF) survival/growth, migration/ invasion, and the ability to degrade cartilage. (A) EPCR mRNA levels in RASFs transfected with EPCR or control small interfering RNA (siRNA) for 24 hours and detected by reverse-transcription real time polymerase chain reaction (PCR). (B) EPCR protein in whole cell lysates of RASFs transfected with EPCR or control siRNA for 72 hours and detected by enzyme-linked immunosorbent assay (ELISA). (C) Viability of RASFs transfected with control or EPCR siRNA or EPCR blocking (RCR252) or non-blocking (RCR92) antibody for 72 hours and detected by colorimetric 3-[4,5-dimethylthiazol-2-yl]-2,5-diphenyl tetrazolium bromide (MTT) and trypan blue exclusion assays. (D) Migration/invasion of RASFs transfected with control or EPCR siRNA for 72 hours. After siRNA transfection for 24 hours, RASFs were embedded into collagen gels at $1 \times 10^{5} \mathrm{cells} / \mathrm{mL}$. After incubation for another 48 hours, collagen gels were stained and cells that had migrated outside of gels were counted (16 gel drops for each treatment). (E) Cartilage degradation by RASFs transfected with control or EPCR siRNA. After 24 hours of siRNA transfection, OA cartilage was co-incubated with RASFs for another 24 hours, and culture medium was then collected for measuring sulphated glycosaminoglycans (sGAGs) by a 1,9-dimethylmethylene blue (DMMB) assay. Cartilage explants were fixed and processed for toluidine blue staining. Data on each graph are shown as mean \pm standard deviation (SD) from four RASF cell lines derived from four RA synovial tissues and analyzed by one-way analysis of variance followed by Tukey's honestly significant difference (HSD) post hoc test (C) or Student $t$ test $(\mathrm{A}, \mathrm{B}, \mathrm{D}, \mathrm{E})$, compared with control siRNA. The images represent one of three experiments Scale bar: $100 \mu \mathrm{m}$. ${ }^{*} P<0.05,{ }^{*} P<0.01$. 
following EPCR siRNA transfection compared with control siRNA (Figure 2D), indicating that suppressing EPCR inhibits RASF migration/invasion. EPCR siRNA transfection also reduced sGAG release, a measure of cartilage degradation [29], into culture media from co-incubated cartilage explants, by more than $40 \%$ (Figure 2E). Toluidine blue staining confirmed that cartilage explants preserved more cartilage when incubated with EPCR siRNA-transfected RASFs when compared with control siRNA (Figure 2E).

Suppressing EPCR reduces MMP-2, IL-1 $\beta$, and cadherin-11 and inactivates NF-KB and MAP kinases

The invasive properties of RASFs are associated with their ability to produce higher levels of inflammatory cytokines and matrix-degrading enzymes and excessive activation of inflammatory signaling molecules such as NF-кB [1,2]. Inhibition of EPCR had no significant effect on matrix-degrading enzyme MMP-9; however, MMP-2 was decreased by $60 \%$ when detected by gelatin zymography (Figure 3A). TNF- $\alpha$ had a minimal effect on MMP-2 but stimulated MMP-9 in both control and EPCR siRNAtransfected cells (Figure 3A). In contrast, APC nearly completely inhibited MMP-9 but stimulated and activated MMP-2. Inhibition of EPCR did not affect the ability of APC to activate MMP-2 (Figure 3A), which is consistent with the ability of APC to directly activate MMP-2 [30].

In response to EPCR siRNA transfection, secretion of $\mathrm{IL}-1 \beta$, the key initiator of inflammation and cartilage

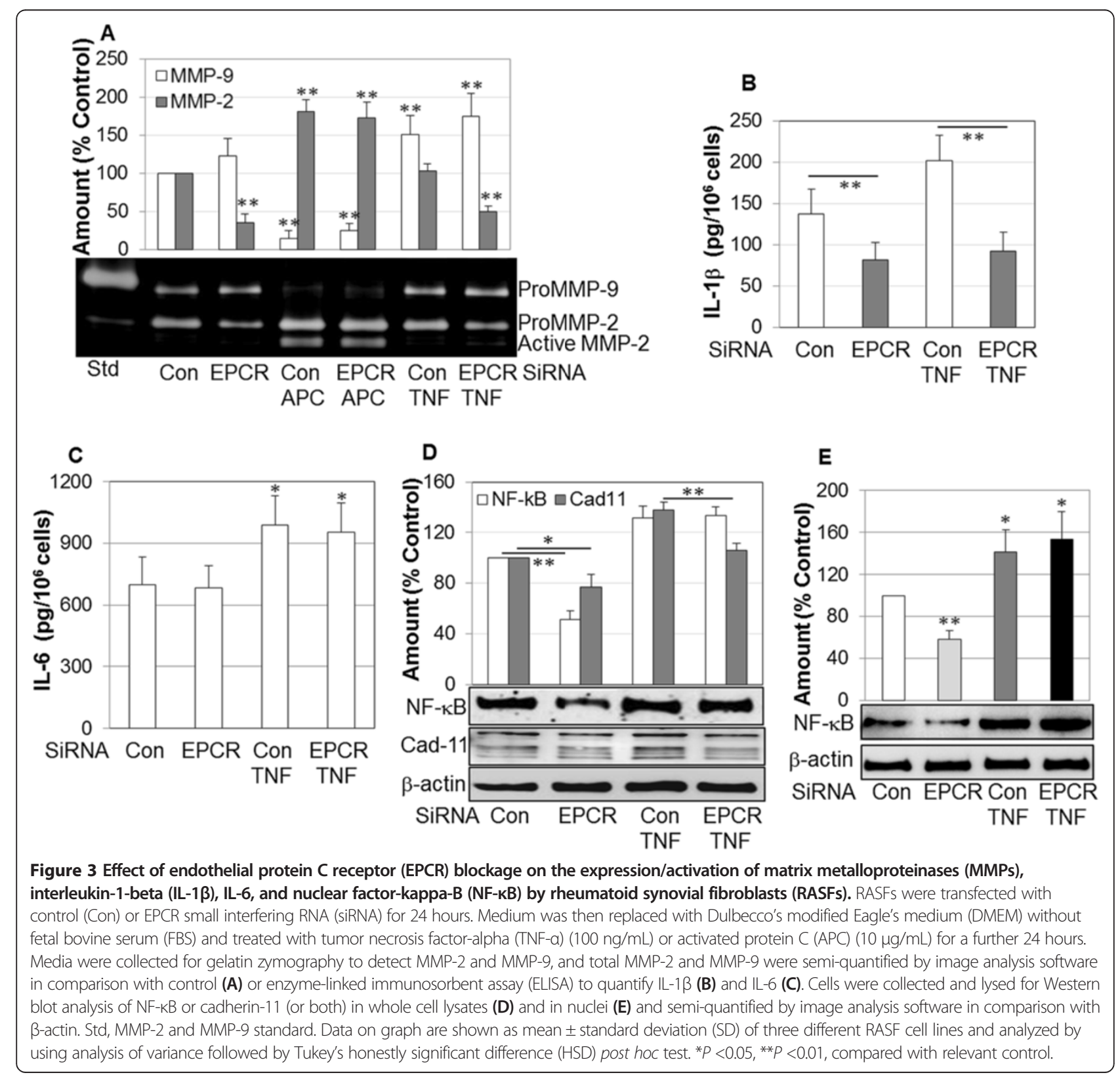


breakdown in RA [31,32], was reduced by approximately $50 \%$ from RASFs (Figure 3B). TNF- $\alpha$ stimulated IL- $1 \beta$ production in control but not in EPCR siRNA-transfected RASFs (Figure 3B). This inhibitory effect was cytokinespecific for IL- $1 \beta$ as silencing EPCR by siRNA had no effect on IL-6 in either control or TNF- $\alpha$-stimulated conditions (Figure 3C).

Silencing EPCR also reduced the activation of NF- $\mathrm{BB}$ in whole cell lysates by $50 \%$ in control but not after TNF- $\alpha$ stimulation (Figure 3D). This was confirmed by the activation of NF- $\mathrm{KB}$ in the nuclear fraction, which displayed a reduction similar to that shown in whole cell lysate (Figure 3E). In addition, cadherin-11, a key molecule that regulates RASF function [33], was significantly suppressed when RASFs were transfected with EPCR siRNA in both control and TNF- $\alpha$-stimulated conditions (Figure 3D).

The expression and activation of mitogen-activated protein (MAP) kinases ERK, p38, and JNK are important in the regulation of RASF survival/growth and inflammation [34-38]. Silencing EPCR inhibited total expression and activation of ERK by more than 50\% (Figure 4A). Although TNF- $\alpha$ stimulated and activated ERK under otherwise basal conditions, it had no effect on ERK when cells were transfected with EPCR siRNA. EPCR siRNA transfection selectively inhibited p38 activation, but not the non-activated form, in the presence or absence of TNF- $\alpha$ (Figure 4B). Silencing EPCR suppressed the activation of JNK in basal conditions but not after TNF- $\alpha$ stimulation (Figure 4C).

\section{sPLA $\mathrm{V}$ co-localizes with EPCR in synovial tissues and blocks APC binding}

The above findings suggest that EPCR promotes inflammation in RA, which is contrary to its well-described anti-inflammatory effects [8]. A recent study showed that $\mathrm{sPLA}_{2} \mathrm{~V}$ inhibits EPCR's cytoprotective function in endothelial cells by preventing APC binding to EPCR [18]. We explored whether $\mathrm{SPLA}_{2} \mathrm{~V}$ was involved in EPCR's inflammatory actions on RASFs. Dual immunofluorescent staining suggested that $\mathrm{SPLA}_{2} \mathrm{~V}$ was colocalized with EPCR in synovial tissues (Figure 5A). In culture, co-immunoprecipitation of cell lysates with antiEPCR antibody followed by Western blotting to detect EPCR generated a band corresponding to EPCR (black arrow) and another band at approximately $60 \mathrm{kD}$ which was the complex of EPCR and $\mathrm{SPLA}_{2} \mathrm{~V}$ (red arrow) (Figure $5 \mathrm{~B}$ ). Further detection of the same membrane with anti-sPLA $\mathrm{A}_{2} \mathrm{~V}$ antibody confirmed that the upper band was the EPCR and $\mathrm{SPLA}_{2} \mathrm{~V}$ complex (Figure $5 \mathrm{~B}$ ). These results indicate that EPCR and $\mathrm{SPLA}_{2} \mathrm{~V}$ can bind together on RASFs. To investigate whether sPLA ${ }_{2} \mathrm{~V}$ could prevent the binding of APC to RASFs, we used two approaches. First, endogenous $\mathrm{sPLA}_{2} \mathrm{~V}$ was suppressed by siRNA for 48 hours, and APC $(10 \mu \mathrm{g} / \mathrm{mL})$ was added to cells for 4 hours. Western blot analysis showed that membrane-associated APC was increased in cells transfected with $\mathrm{SPLA}_{2} \mathrm{~V}$ siRNA when compared with cells transfected with control siRNA (Figure 5C). Second, when RASFs were pre-incubated with recombinant $\mathrm{SPLA}_{2} \mathrm{~V}$ before the addition of APC, there was markedly
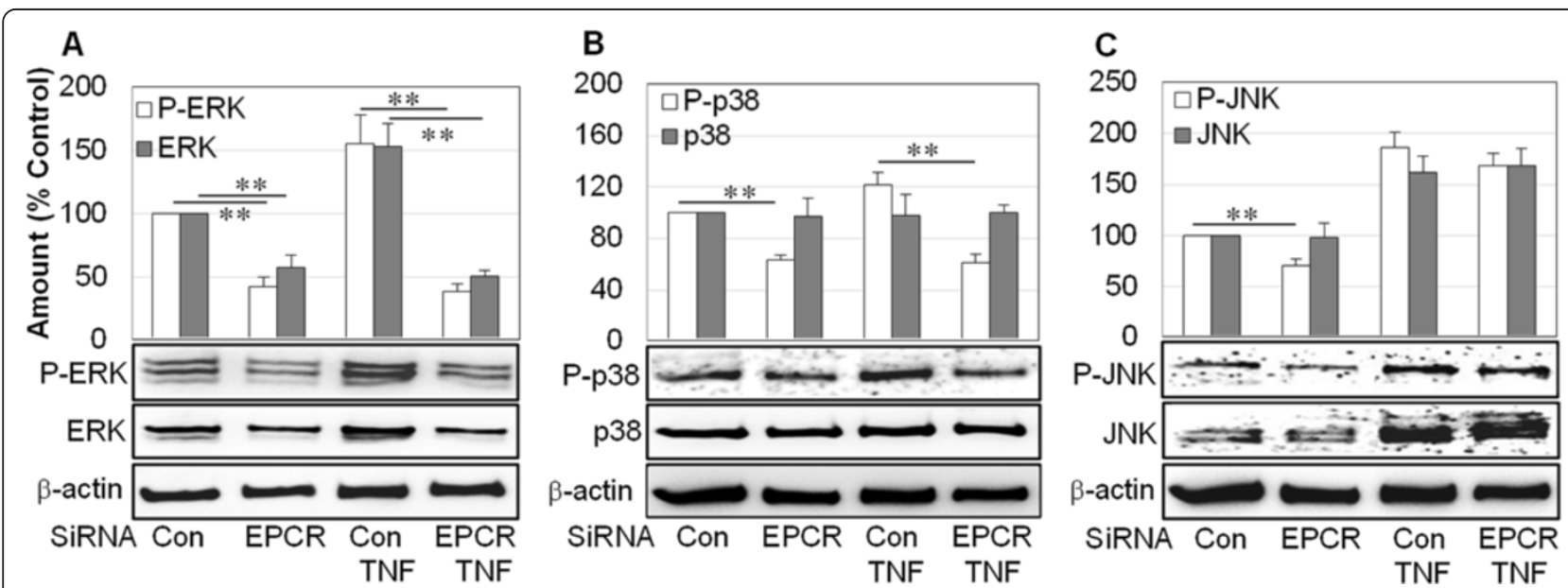

Figure 4 Effect of endothelial protein $C$ receptor (EPCR) blockage on the expression/activation of mitogen-activated protein (MAP) kinase ERK, p38, and JNK in rheumatoid synovial fibroblasts (RASFs). RASFs were transfected with control (Con) or EPCR small interfering RNA (siRNA) for 24 hours. Medium was then replaced with Dulbecco's modified Eagle's medium (DMEM) without fetal bovine serum (FBS) and treated with tumor necrosis factor-alpha (TNF-a) $(100 \mathrm{ng} / \mathrm{mL})$ for a further 24 hours. Cells were collected and lysed for Western blot analysis and semi-quantified by image analysis software to measure ERK and P-ERK (A), p38 and P-p38 (B), and JNK and P-JNK (C); $\beta$-actin was included as an internal standard. The gel images represent one of three experiments. Data on graphs are shown as mean \pm standard deviation (SD) of three different RASF cell lines and were analyzed by analysis of variance followed by Tukey's honestly significant difference (HSD) post hoc test. ${ }^{*} P<0.01$. Relative comparisons are indicated by horizontal lines. 

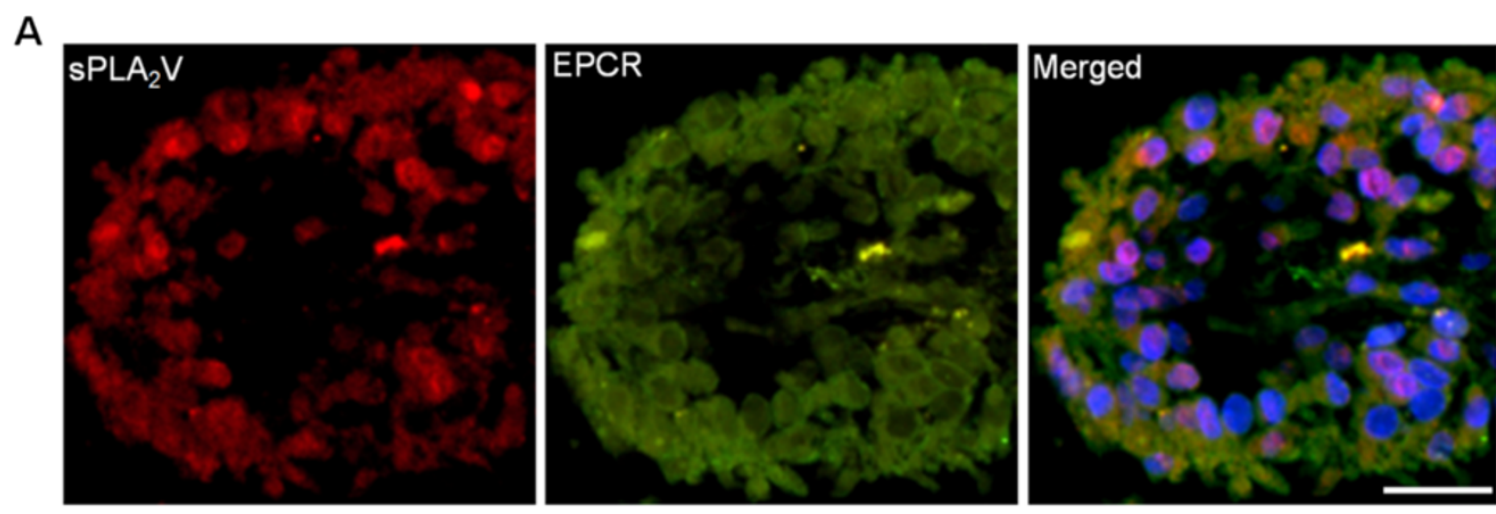

B

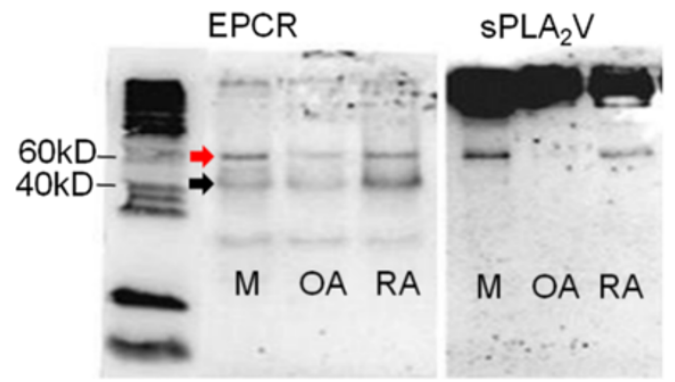

C

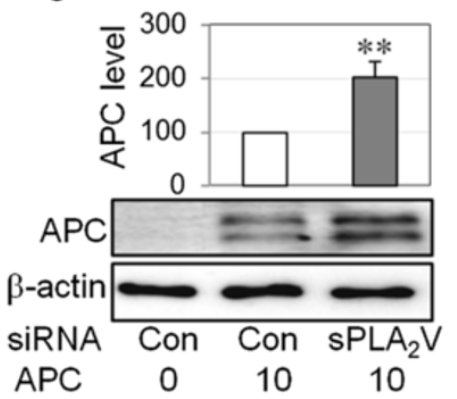

D

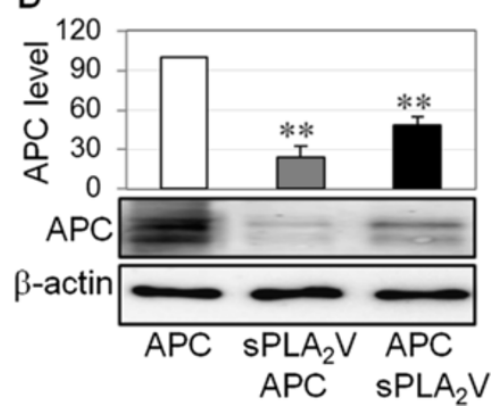

Figure 5 Group V secretory phospholipase $A_{2}\left(s P L A_{2} V\right)$ is co-localized with endothelial protein $C$ receptor (EPCR) and blocks activated protein C (APC) binding to rheumatoid synovial fibroblasts (RASFs). (A) SPLA $V$ was co-localized with EPCR in rheumatoid arthritis (RA) synovial tissue, detected by immunofluorescent staining. Nuclei were counterstained by 4'-6-diamidino-2-phenylindole (DAPI) (blue). Scale bar: $50 \mu \mathrm{m}$. (B) SPLA 2 and EPCR expression by MCF-7 cells (M, used as a positive control), RASFs (RA), or OASFs (OA), detected by immunoprecipitation using anti-EPCR antibody and followed by Western blot using anti-EPCR or SPLA $\mathrm{A}_{2}$ antibody under non-reducing conditions. Red arrow indicates EPCR and $s P L A_{2} V$ complex. Black arrow indicates EPCR. (C) APC in whole cell lysates of RASFs transfected with small interfering RNA (siRNA) for control (Con) or sPLA 2 siRNA for 48 hours and treated with APC $(10 \mu \mathrm{g} / \mathrm{mL})$ for 24 hours and detected by Western blot. (D) APC in the whole cell lysates of RASFs treated with recombinant APC $(2 \mu \mathrm{g} / \mathrm{mL})$, recombinant sPLA $\mathrm{A}_{2} \mathrm{~V}(2 \mu \mathrm{g} / \mathrm{mL})$ for first 30 minutes then APC (sPLA $V+$ APC) or APC for first 30 minutes then $s P L A_{2} V\left(A P C+s P L A_{2} V\right)$ for 4 hours, detected by Western blot. The images represent one of three different experiments using three different RASF cell lines. Data on (C) and (D) were semi-quantified by image analysis software in comparison with $\beta$-actin, expressed as a percentage of control and shown as mean \pm standard deviation (SD) $(n=4)$ and analyzed by one-way analysis of variance followed by Tukey's honestly significant difference (HSD) post hoc test. ${ }^{* *} P<0.01$.

less cell-associated APC compared with APC alone or with addition of APC prior to $\mathrm{SPLA}_{2} \mathrm{~V}$ (Figure 5D). These data suggest that $\mathrm{SPLA}_{2} \mathrm{~V}$ prevents $\mathrm{APC}$ binding to RASFs.

\section{sPLA 2 promotes the aggressive properties of RASFs via EPCR}

To examine whether $\mathrm{SPLA}_{2} \mathrm{~V}$ regulates the aggressive properties of RASFs, cell viability and cartilage degradation were examined after transfection with $\mathrm{SPLA}_{2} \mathrm{~V}$ siRNA. Silencing $\mathrm{SPLA}_{2} \mathrm{~V}$ significantly reduced cell viability by more than $20 \%$ (Figure $6 \mathrm{~A}$ ), cartilage degradation by more than $40 \%$ (Figure $6 \mathrm{~B}$ ), and IL-1 $\beta$ production by more than $40 \%$ (Figure $6 \mathrm{C}$ ). Furthermore, recombinant $\mathrm{sPLA}_{2} \mathrm{~V}$ at $100 \mathrm{ng} / \mathrm{mL}$ promoted RASF-mediated cartilage degradation by approximately 30\% (Figure 6D) but did not significantly raise RASF viability after 72 hours of treatment (data not shown). To investigate whether
sPLA $_{2} \mathrm{~V}$-associated aggressive properties of RASFs are mediated by EPCR, RASFs were transfected with EPCR siRNA and stimulated with recombinant $\mathrm{SPLA}_{2} \mathrm{~V}$. sPLA $2 \mathrm{~V}$ significantly increased RASF-mediated sGAG release and NF- $\kappa \mathrm{B}$ activation in control cells (Figure 6E and F); however, there was a $40 \%$ reduction in $\mathrm{SPLA}_{2} \mathrm{~V}$-stimulated sGAG release and $45 \%$ reduction in $\mathrm{SPLA}_{2} \mathrm{~V}$-stimulated NF- $\kappa B$ activation in EPCR siRNA-transfected cells (Figure 6E and F).

Taken together, these data suggest that $\mathrm{sPLA}_{2} \mathrm{~V}$ is mainly responsible for RASF-mediated cartilage degradation and inflammation associated with overexpression of EPCR.

\section{Discussion}

SFs are key effectors in the pathogenesis of RA. In this study, we have demonstrated that RASFs express higher 


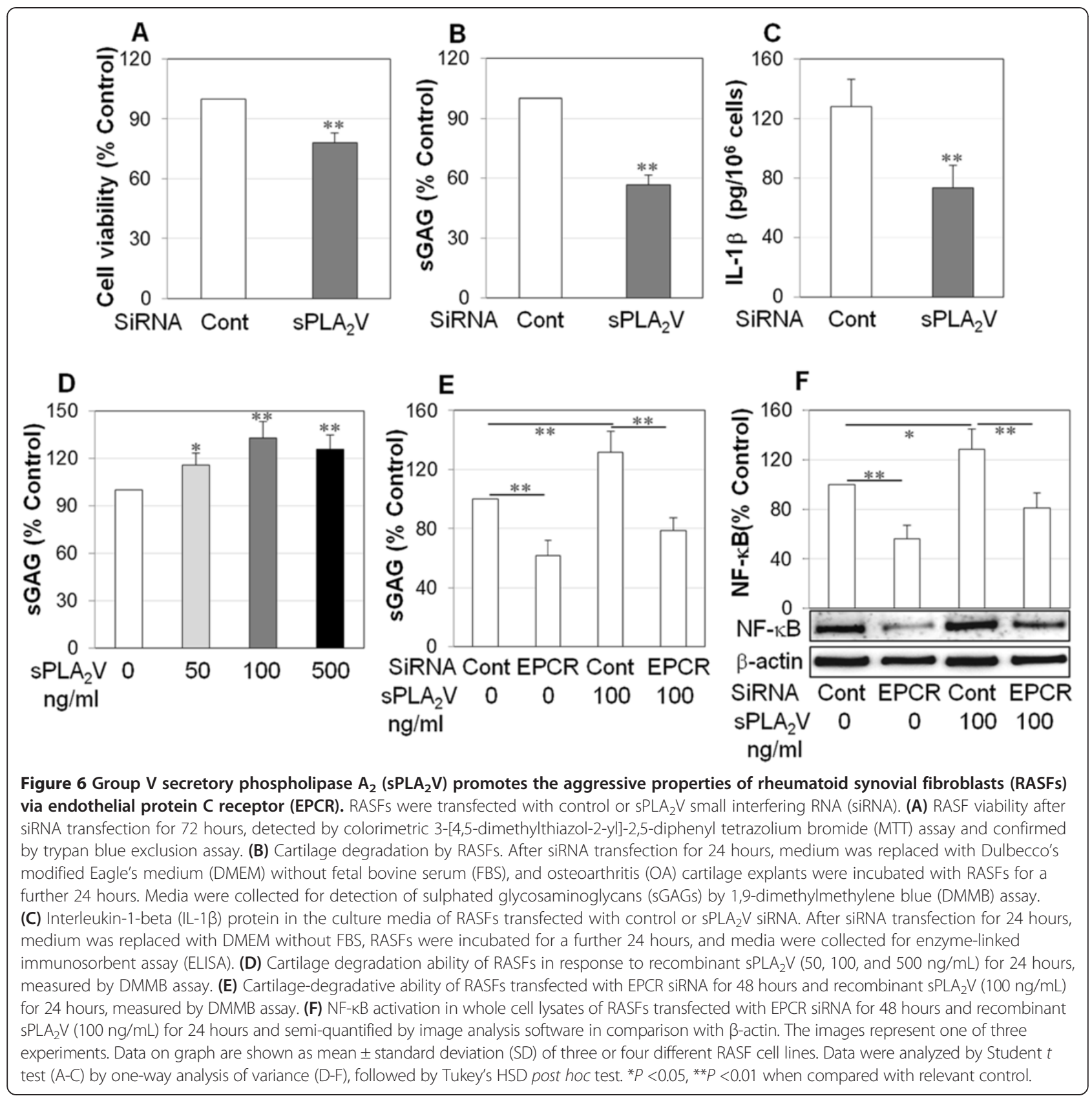

levels of EPCR than OASFs. In contrast to its conventional anti-inflammatory effects, EPCR expressed by RASFs was associated with increased invasiveness and inflammatory responses of these cells. Further investigation revealed that $\mathrm{sPLA}_{2} \mathrm{~V}$ is co-localized with EPCR, prevents APC from interacting with EPCR, and drives EPCR-associated invasiveness, inflammation, and cartilage degradation.

EPCR plays a critical role in augmenting PC activation and mediating the anti-inflammatory and cytoprotective functions of the PC pathway [39]. In this study, however, EPCR expression was associated with the destructive effects of RASFs. Suppressing EPCR decreased RASF viability, invasion, and cartilage degradation ability through inhibition of inflammatory cytokine IL-1 $\beta$. In RA, IL-1 $\beta$ stimulates the production of MMPs and the maturation of osteoclasts and ultimately promotes cartilage breakdown [31,32] and the development of bone erosion $[40,41]$. In synovium, IL- $1 \beta$ is a major activator of SFs by promoting the activation of NF- $\mathrm{kB}$ and MAP kinases [1]. Moreover, IL-1 $\beta$, but not TNF- $\alpha$, can markedly induce $\mathrm{sPLA}_{2} \mathrm{~V}$ production by SFs [21].

Suppression of EPCR also markedly reduced the expression and activation of MAP kinases which regulate 
cell survival, apoptosis, viability, cellular stress, and inflammatory responses. The three major classes of MAP kinases-ERK, p38, and JNK-are all enhanced in RA synovial tissues $[37,38]$. Survival/growth of RASFs is mediated by ERK [35], which plays an important role in the maintenance of RA by promoting pannus formation [34]. JNK activation is required for the regulation of collagenase production by SFs [35,36]. ERK and JNK activation predict development of erosive disease in early arthritis [35,36]. Our results suggest that EPCR promotes RASF viability and inflammation via activation of MAP kinases. Inhibition of NF- $\mathrm{kB}$ and cadherin-11 by suppression of EPCR also likely contributes to reduced invasion and cartilage degradation by RASFs [33,42]. Our data also show that suppressing EPCR in RASFs inhibits production of MMP2, a function of EPCR that is present in endothelial cells [43]. Although MMP-2 may promote cartilage degradation, it suppresses the development of inflammatory joint disease in a mouse arthritis model [44]. MMP-2 is constitutively expressed by RASFs, and the exact role of MMP-2 activity by these cells is still unclear.

Our unexpected finding that EPCR is not cytoprotective in RASFs has precedent in cancer cells. EPCR increases cell migration and invasion of breast cancer cells in vitro [9] and is a possible biomarker of ovarian cancer onset [10]. EPCR also promotes metastasis and correlates with clinical outcome in lung adenocarcinoma [11]. However, vascular wall EPCR inhibits cancer cell adhesion and transmigration [45]. The reasons for these contradictory findings are unclear but may reflect the different regulatory mechanisms of EPCR in different cell types and tissues.

EPCR can be regulated by proteolytic release from the cell surface to form sEPCR $[12,13]$. sEPCR binds PC to inhibit APC generation or binds APC to block the protective function of APC [14]. Pro-inflammatory cytokines IL$1 \beta$ and TNF- $\alpha$ enhance EPCR shedding from the endothelial cell surface [16,46]. Accordingly, higher levels of sEPCR have been reported in patients with systemic inflammatory diseases $[15,17,47]$. sEPCR produced by ovarian cancer cells is a possible biomarker of cancer onset [10] and is likely to be a biomarker of cancer-associated hypercoagulability in human hematologic malignancies [48]. However, in the present study, we found that cellassociated EPCR is three times higher in cultured RASFs than in OASFs and that there is no difference in SEPCR, either in cultured supernatants of OASFs and RASFs or in synovial fluids from patients with OA and RA (Figure 1). These data suggest that it is not sEPCR, but the membrane bound form, that exerts the inflammatory and cartilage-degradative actions of RASFs. This destructive property of EPCR differs from its cytoprotective actions in other settings [49]. The present study explored the reasons for this diametrical role of EPCR in RA. Although
RASFs express PC/APC, neither silencing endogenous PC by siRNA nor adding recombinant APC significantly changed RASF viability (Additional file 1 shows this in more detail), indicating that this function of EPCR is not due to PC/APC. We found that this paradox may be explained by the actions of $\mathrm{sPA}_{2} \mathrm{~V}$, which generates bioactive lipids LysoPCh and PAF. These two lipids can substitute for PCh, which normally resides in the deep groove of EPCR and is required for normal EPCR function. When LysoPCh or PAF substitutes for PCh, they impair the ability of EPCR to interact with PC or APC, thus inhibiting EPCR's cytoprotective function in endothelial cells [18].

In the present study, RASFs expressed high levels of $\mathrm{SPLA}_{2} \mathrm{~V}$, which promoted the aggressive properties of RASFs. Suppressing endogenous $\mathrm{SPLA}_{2} \mathrm{~V}$ reduced RASF viability, cartilage degradation ability, and IL-1 $\beta$, whereas recombinant $\mathrm{SPLA}_{2} \mathrm{~V}$ enhanced RASF-mediated cartilage degradation and NF- $\mathrm{kB}$ activation (Figure 6). Further study using dual immunostaining and co-immunoprecipitation indicated that $\mathrm{sPLA}_{2} \mathrm{~V}$ and EPCR are spatially associated with each other on RASFs. We found that $\mathrm{sPA}_{2} \mathrm{~V}$ not only blocks APC binding to RASFs but also uses EPCR to promote its inflammatory effects on RASFs. This was evidenced by the fact that suppressing endogenous ${ }_{\mathrm{SPLA}} \mathrm{V}$ enhanced but that recombinant $\mathrm{SPLA}_{2} \mathrm{~V}$ inhibited APC binding to RASFs (Figure 5). Moreover, suppression of EPCR reduced the stimulatory effect of $\mathrm{SPLA}_{2} \mathrm{~V}$ on cartilage degradation and NF- $\mathrm{kB}$ activation by RASFs (Figure 6). We propose that, similar to its effect on endothelial cells [18], sPLA2V impairs the ability of EPCR to interact with APC in RASFs, inhibiting EPCR's cytoprotective function.

\section{Conclusions}

In summary, this study demonstrated that elevated EPCR promotes the inflammatory responses and invasiveness of RASFs, which are likely driven by $\mathrm{sPA}_{2} \mathrm{~V}$. These results provide new insights into the mechanisms underlying SFmediated joint inflammation in RA and may inspire new targeted therapeutic approaches.

\section{Additional file}

Additional file 1: Figure S1. Protein C/activated protein $C(P C / A P C)$ expression and its effect on rheumatoid synovial fibroblast (RASF) viability.

\section{Abbreviations}

APC: activated protein C; DMEM: Dulbecco's modified Eagle's medium; ELISA: enzymelinked immunosorbent assay; EPCR: endothelial protein C receptor; FBS: fetal bovine serum; IL: interleukin; IP: immunoprecipitation; LysoPCh: lysophosphotidylcholine; MAP: mitogen-activated protein; MMP: matrix metalloproteinase; MTT: colorimetric 3-[4,5-dimethylthiazol-2-yl]-2,5-diphenyl tetrazolium bromide; NF-KB: nuclear factor-kappa-B; OA: osteoarthritis; PAF: platelet-activating factor; PC: protein C; RA: rheumatoid arthritis; RASF: rheumatoid synovial fibroblast; sEPCR: soluble endothelial protein C receptor; SF: synovial fibroblast; sGAG: sulphated glycosaminoglycan; siRNA: small interfering RNA; ${ }_{S P L A} V$ : group $V$ secretory phospholipase $A_{2} ;$ TNF-a: tumor necrosis factor-alpha. 


\section{Competing interests}

The authors declare that they have no competing interests.

\section{Authors' contributions}

MX participated in conception and design, data acquisition, collection and analysis, manuscript writing, and final approval of the manuscript. KS, KM, JL, Y-KAC, and LM participated in data acquisition, collection and analysis, and critical revision and final approval of the manuscript. VH, CBL, and MT participated in data acquisition, analysis, and critical revision and final approval of the manuscript. CJJ participated in data acquisition, collection and analysis, manuscript writing, and critical revision and final approval of the manuscript. All authors read and approved the final manuscript.

\section{Acknowledgments}

We wish to thank Benjamin Chan from Raymond Purves Laboratory for his help in 1,9-dimethylmethylene blue (DMMB) assay. Financial support was provided by National Health and Medical Research Council, Royal North Shore Hospital Staff Specialist Trust fund, Arthritis Australia, and Henry Langley Research Fellowship.

\section{Author details}

${ }^{1}$ Sutton Arthritis Research Laboratories, The University of Sydney at Royal North Shore Hospital, Pacific Highway, St Leonards, NSW 2065, Australia. ${ }^{2}$ Department of Rheumatology, The University of Sydney at Royal North Shore Hospital, Pacific Highway, St Leonards, NSW 2065, Australia. ${ }^{3}$ Raymond Purves Research Laboratory, The University of Sydney at Royal North Shore Hospital, Pacific Highway, St Leonards, NSW 2065, Australia. ${ }^{4}$ Department of Surgery, Kolling Institute of Medical Research, The University of Sydney at Royal North Shore Hospital, Pacific Highway, St Leonards, NSW 2065, Australia.

Received: 11 June 2013 Accepted: 28 January 2014

Published: 5 February 2014

\section{References}

1. Noss EH, Brenner MB: The role and therapeutic implications of fibroblastlike synoviocytes in inflammation and cartilage erosion in rheumatoid arthritis. Immunol Rev 2008, 223:252-270.

2. Huber LC, Distler O, Tarner I, Gay RE, Gay S, Pap T: Synovial fibroblasts: key players in rheumatoid arthritis. Rheumatology 2006, 45:669-675.

3. Muller-Ladner U, Kriegsmann J, Franklin BN, Matsumoto S, Geiler T, Gay RE, Gay S: Synovial fibroblasts of patients with rheumatoid arthritis attach to and invade normal human cartilage when engrafted into SCID mice. Am J Pathol 1996, 149:1607-1615.

4. Lefèvre S, Knedla A, Tennie C, Kampmann A, Wunrau C, Dinser R, Korb A, Schnäker EM, Tarner IH, Robbins PD, Evans CH, Stürz H, Steinmeyer J, Gay S, Schölmerich J, Pap T, Müller-Ladner U, Neumann E: Synovial fibroblasts spread rheumatoid arthritis to unaffected joints. Nat Med 2009, 15:1414-1420.

5. Fukudome K, Esmon CT: Identification, cloning, and regulation of a novel endothelial cell protein c activated protein c receptor. J Biol Chem 1994, 269:26486-26491.

6. Bouwens EA, Mosnier LO: EPCR encryption induces cellular APC resistance. Blood 2012, 119:2703-2705.

7. Xue M, March L, Sambrook PN, Fukudome F, Jackson CJ: Endothelia protein $C$ receptor is over-expressed in rheumatoid arthritic (RA) synovium and mediates the anti-inflammatory effects of activated protein C in RA monocytes. Ann Rheum Dis 2007, 66:1574-1580.

8. Esmon CT: Structure and functions of the endothelial cell protein C receptor. Crit Care Med 2004, 32:S298-S301

9. Gramling MW, Beaulieu LM, Church FC: Activated protein C enhances cell motility of endothelial cells and MDA-MB-231 breast cancer cells by intracellular signal transduction. Exp Cell Res 2010, 316:314-328.

10. Ducros E, Mirshahi S, Azzazene D, Camilleri-Broët S, Mery E, Al Farsi H, Althawadi H, Besbess S, Chidiac J, Pujade-Lauraine E, Therwath A, Soria J, Mirshahi M: Endothelial protein $C$ receptor expressed by ovarian cancer cells as a possible biomarker of cancer onset. Int J Oncol 2012, 41:433-440.

11. Antón I, Molina E, Luis-Ravelo D, Zandueta C, Valencia K, Ormazabal C, Martínez-Canarias S, Perurena N, Pajares MJ, Agorreta J, Montuenga LM, Segura V, Wistuba II, De Las Rivas J, Hermida J, Lecanda F: Receptor of activated protein C promotes metastasis and correlates with clinical outcome in lung adenocarcinoma. Am J Respir Crit Care Med 2012, 186:96-105.

12. $\mathrm{Xu} J, \mathrm{Qu} \mathrm{D}$, Esmon NL, Esmon CT: Metalloproteolytic release of endothelial cell protein C receptor. J Biol Chem 2000, 275:6038-6044.

13. Qu D, Wang Y, Esmon NL, Esmon CT: Regulated endothelial protein C receptor shedding is mediated by tumor necrosis factor-alpha converting enzyme/ADAM17. J Thromb Haemost 2007, 5:395-402.

14. Liaw PC, Neuenschwander PF, Smirnov MD, Esmon CT: Mechanisms by which soluble endothelial cell protein $C$ receptor modulates protein $C$ and activated protein C function. J Biol Chem 2000, 275:5447-5452.

15. Kurosawa S, Stearnskurosawa DJ, Carson CW, Dangelo A, Dellavalle P, Esmon CT: Plasma levels of endothelial cell protein c receptor are elevated in patients with sepsis and systemic lupus erythematosus - lack of correlation with thrombomodulin suggests involvement of different pathological processes. Blood 1998, 91:725-727.

16. Gu JM, Katsuura Y, Ferrell GL, Grammas P, Esmon CT: Endotoxin and thrombin elevate rodent endothelial cell protein $C$ receptor mRNA levels and increase receptor shedding in vivo. Blood 2000, 95:1687-1693.

17. Bilgic A, Ozdemir FN, Bayraktar N, Karakus S, Sasak G, Arat Z, Sezer S, Haberal $M$ : Soluble endothelial protein $C$ receptor: influence on arteriovenous fistula thrombosis development in hemodialysis patients. Am J Nephrol 2007, 27:366-372.

18. Lopez-Sagaseta J, Puy C, Tamayo I, Allende M, Cervero J, Velasco SE, Esmon CT, Montes R, Hermida J: sPLA2-V inhibits EPCR anticoagulant and antiapoptotic properties by accommodating lysophosphatidylcholine or PAF in the hydrophobic groove. Blood 2012, 119:2914-2921.

19. Pruzanski W, Vadas P: Phospholipase A2-a mediator between proximal and distal effectors of inflammation. Immunol Today 1991, 12:143-146.

20. Leistad L, Feuerherm AJ, Ostensen M, Faxvaag A, Johansen B: Presence of secretory group Ila and V phospholipase A2 and cytosolic group IValpha phospholipase A2 in chondrocytes from patients with rheumatoid arthritis. Clin Chem Lab Med 2004, 42:602-610.

21. Masuda S, Murakami M, Komiyama K, Ishihara M, Ishikawa Y, Ishii T, Kudo I: Various secretory phospholipase A2 enzymes are expressed in rheumatoid arthritis and augment prostaglandin production in cultured synovial cells. FEBS J 2005, 272:655-672

22. Altman R, Asch E, Bloch D, Bole G, Borenstein D, Brandt K, Christy W, Cooke TD, Greenwald R, Hochberg M: Development of criteria for the classification and reporting of osteoarthritis, Classification of osteoarthritis of the knee. Diagnostic and Therapeutic Criteria Committee of the American Rheumatism Association. Arthritis Rheum 1986, 8:49.

23. Arnett FC, Edworthy SM, Bloch DA, McShane DJ, Fries JF, Cooper NS: The American Rheumatism Association 1987 revised criteria for the classification of rheumatoid arthritis. Arthritis Rheum 1988, 31:315-324.

24. Herron GS, Banda MJ, Clark EJ, Gavrilovic J, Werb Z: Secretion of metalloproteinases by stimulated capillary endothelial cells. II. Expression of collagenase and stromelysin activities is regulated by endogenous inhibitors. J Biol Chem 1986, 261:2814-2818.

25. Korff $\mathrm{T}$, Augustin HG: Tensional forces in fibrillar extracellular matrices control directional capillary sprouting. J Cell Sci 1999, 112:3249-3258.

26. Xue M, Chan YK, Shen K, Dervish S, March L, Sambrook PN, Jackson CJ: Protease-activated receptor 2 , rather than protease-activated receptor 1 , contributes to the aggressive properties of synovial fibroblasts in rheumatoid arthritis. Arthritis Rheum 2012, 64:88-98.

27. Schmitz N, Laverty S, Kraus VB, Aigner T: Basic methods in histopathology of joint tissues. Osteoarthritis Cartilage 2010, 18:S113-S116.

28. Farndale RW, Buttle DJ, Barrett AJ: Improved quantitation and discrimination of sulphated glycosaminoglycans by use of dimethylmethylene blue. Biochim Biophys Acta 1986, 883:173-177.

29. Korb-Pap A, Stratis A, Mühlenberg K, Niederreiter B, Hayer S, Echtermeyer F, Stange R, Zwerina J, Pap T, Pavenstädt H, Schett G, Smolen JS, Redlich K: Early structural changes in cartilage and bone are required for the attachment and invasion of inflamed synovial tissue during destructive inflammatory arthritis. Ann Rheum Dis 2012, 71:1004-1011.

30. Nguyen M, Arkell J, Jackson CJ: Activated protein C directly activates human endothelial gelatinase A. J Biol Chem 2000, 275:9095-9098.

31. Goldring SR: Pathogenesis of bone and cartilage destruction in rheumatoid arthritis. Rheumatology (Oxford) 2003, 42:11-16.

32. Pretzel D, Pohlers D, Weinert S, Kinne RW: In vitro model for the analysis of synovial fibroblast-mediated degradation of intact cartilage. Arthritis Res Ther 2009, 11:R25. 
33. Lee DM, Kiener HP, Agarwal SK, Noss EH, Watts GF, Chisaka O, Takeichi M, Brenner MB: Cadherin-11 in synovial lining formation and pathology in arthritis. Science 2007, 315:1006-1010.

34. Schett G, Tohidast-Akrad M, Smolen JS, Schmid BJ, Steiner CW, Bitzan P, Zenz P, Redlich K, Xu Q, Steiner G: Activation, differential localization, and regulation of the stress-activated protein kinases, extracellular signalregulated kinase, c-JUN N-terminal kinase, and p38 mitogen-activated protein kinase, in synovial tissue and cells in rheumatoid arthritis. Arthritis Rheum 2000, 43:2501-2512.

35. Zhang Y, Dong J, He P, Li W, Zhang Q, Li N, Sun T: Genistein inhibit cytokines or growth factor-induced proliferation and transformation phenotype in fibroblast-like synoviocytes of rheumatoid arthritis. Inflammation 2012, 35:377-387.

36. Han Z, Boyle DL, Chang L, Bennett B, Karin M, Yang L, Manning AM, Firestein GS: c-Jun $\mathrm{N}$-terminal kinase is required for metalloproteinase expression and joint destruction in inflammatory arthritis. J Clin Invest 2001, 108:73-81.

37. de Launay D, van de Sande MG, de Hair MJ, Grabiec AM, van de Sande GP, Lehmann KA, Wijbrandts CA, van Baarsen LG, Gerlag DM, Tak PP, Reedquist KA: Selective involvement of ERK and JNK mitogen-activated protein kinases in early rheumatoid arthritis (1987 ACR criteria compared to 2010 ACR/EULAR criteria): a prospective study aimed at identification of diagnostic and prognostic biomarkers as well as therapeutic targets. Ann Rheum Dis 1987, 2012:415-423.

38. Gortz B, Hayer S, Tuerck B, Zwerina J, Smolen JS, Schett G: Tumour necrosis factor activates the mitogen-activated protein kinases p38alpha and ERK in the synovial membrane in vivo. Arthritis Res Ther 2005, 7:R1140-R1147.

39. Gleeson EM, O'Donnell JS, Preston RJ: The endothelial cell protein C receptor: cell surface conductor of cytoprotective coagulation factor signaling. Cell Mol Life Sci 2012, 69:717-726.

40. Joosten LA, Helsen MM, Saxne T, van De Loo FA, Heinegard D, van Den Berg WB: IL-1 alpha beta blockade prevents cartilage and bone destruction in murine type II collagen-induced arthritis, whereas TNF-alpha blockade only ameliorates joint inflammation. J Immunol 1999, 163:5049-5055.

41. Trebec-Reynolds DP, Voronov I, Heersche JN, Manolson MF: IL-1alpha and IL-1 beta have different effects on formation and activity of large osteoclasts. J Cell Biochem 2010, 109:975-982.

42. Criswell LA: Gene discovery in rheumatoid arthritis highlights the CD40/ NF-kappaB signaling pathway in disease pathogenesis. Immunol Rev 2010, 233:55-61.

43. Xue M, Minhas N, Chow SO, Dervish S, Sambrook PN, March L, Jackson CJ: Endogenous protein $\mathrm{C}$ is essential for the functional integrity of human endothelial cells. Cell Mol Life Sci 2010, 67:1537-1546.

44. Itoh T, Matsuda H, Tanioka M, Kuwabara K, Itohara S, Suzuki R: The role of matrix metalloproteinase- 2 and matrix metalloproteinase- 9 in antibodyinduced arthritis. J Immunol 2002, 169:2643-2647.

45. Bezuhly M, Cullen R, Esmon CT, Morris SF, West KA, Johnston B, Liwski RS: Role of activated protein $C$ and its receptor in inhibition of tumor metastasis. Blood 2009, 113:3371-3374.

46. Menschikowski M, Hagelgans A, Eisenhofer G, Siegert G: Regulation of endothelial protein $\mathrm{C}$ receptor shedding by cytokines is mediated through differential activation of MAP kinase signaling pathways. Exp Cell Res 2009, 315:2673-2682.

47. Wu J, Zhou Z, Ye S, Dai H, Ma L, Xu X, Li X: Detection of soluble endothelial protein $C$ receptor (sEPCR) in patients with CHD, DM and SLE. Zhonghua XueYeXueZa Zhi 2000, 21:472-474.

48. Ducros E, Mirshahi SS, Faussat AM, Mirshahi P, Dimicoli S, Tang R, Pardo J, Ibrahim J, Marie JP, Therwath A, Soria J, Mirshahi M: Soluble endothelial protein C receptor (SEPCR) is likely a biomarker of cancer-associated hypercoagulability in human hematologic malignancies. Cancer Med 2012, 1:261-267.

49. Montes R, Puy C, Molina E, Hermida J: Is EPCR a multi-ligand receptor? Pros and cons. Thromb Haemost 2012, 107:815-826.

\section{doi:10.1186/ar4473}

Cite this article as: Xue et al: Endothelial protein C receptor-associated invasiveness of rheumatoid synovial fibroblasts is likely driven by group $V$ secretory phospholipase A2. Arthritis Research \& Therapy 2014 16:R44.

\section{Submit your next manuscript to BioMed Central and take full advantage of:}

- Convenient online submission

- Thorough peer review

- No space constraints or color figure charges

- Immediate publication on acceptance

- Inclusion in PubMed, CAS, Scopus and Google Scholar

- Research which is freely available for redistribution

Submit your manuscript at www.biomedcentral.com/submit
C Biomed Central 